\title{
VIABILIDADE ECONÔMICA NA ESCOLHA ENTRE INVESTIR NO USO DE GÁS OU GASOLINA PARA MOTORISTAS UBER NO CEARÁ
}

\author{
Breno Gomes Oliveira (UFC) brenoliveira.ec@gmail.com \\ Francisco Hernelde Araújo Júnior (UFC) jhernelde@gmail.com \\ Júlio César Machado de Magalhães (UFC) julio_seguro@ hotmail.com \\ Marcos César Cabral da Silva Júnior (UFC) marcos-jr-@ hotmail.com \\ Vinicius dos Santos Soares (UFC) viniciussantos8@outlook.com \\ Maxweel Veras Rodrigues (UFC) maxweelveras@gmail.com
}

\section{Resumo}

Em meio à crise financeira e a alta taxa de desemprego no país, uma das principais soluções, para milhares de pessoas, foi dirigir por aplicativo em tempo integral ou nas horas vagas. Porém, nos últimos anos, no Brasil, o preço da gasolina aumentou de forma considerável e muitas pessoas começaram a aderir outra forma de combustível, como: o gás natural veicular (GNV), pois este possui uma autonomia maior se comparado à gasolina, todavia há um preço a mais para sua instalação e manutenção. Com isso, o objetivo desse trabalho é avaliar a viabilidade econômica da implantação do GNV como forma alternativa à gasolina, para carros populares que serão usados por motoristas de aplicativo, no caso do estudo será o da Uber, no estado do Ceará. A pesquisa possui caráter exploratório, com informações advindas de pesquisas bibliográficas e de documentos. Diante da análise de investimento, esta terá como indicadores: o payback, Valor Presente Líquido (VPL) e a Taxa Interna de Retorno (TIR). Ao final do estudo, pode-se observar que a troca do uso da gasolina pelo gás natural veicular, mesmo com os gastos a mais deste último, é válida e resultará em um maior ganho econômico para os motoristas do aplicativo.

Palavras-chave: Análise de investimentos. Combustível. UBER

\section{Introdução}

Desde o início do funcionamento do aplicativo Uber no Brasil, em 2014, o número de cidades em que o aplicativo está presente, de usuários, assim como o número de motoristas vinculados ao mesmo tem crescido bastante. Tais valores, divulgados pela própria empresa, e atualizados em setembro de 2018, reafirmam o que foi dito anteriormente: o Uber está presente em mais de 
100 cidades brasileiras; possui mais de 600 mil motoristas parceiros e mais de 22 milhões de usuários no Brasil. Além disso, o transporte remunerado privado individual de passageiros foi regularizado em março de 2018 através da Lei no 13.640.

É válido citar que esses valores, tão relevantes, possuem motivações diversas. Tal aplicativo, muitas vezes é usado como uma forma de superar o elevado índice de desemprego no país. Segundo dados divulgados pelo IBGE - Instituto Brasileiro de Geografia e Estatística - com valores referentes até final de setembro de 2019, a taxa de desocupados no Brasil é cerca de $11,8 \%$, sendo uma soma de 12,5 milhões de pessoas. Acrescido a isso, há o desconforto da estrutura do transporte público nacional, a demora e superlotação dos meios de transporte, além da insegurança, que propiciam assim o aumento do uso de aplicativos de transporte individual. Tendo como base um ranking feito pelo instituto de pesquisa Expert Market, sobre sistemas de transportes em diversas partes do mundo, várias cidades brasileiras encontravam-se em posições ruins, reafirmando ainda mais tal realidade.

Ademais, outro ponto que deve ser mencionado é o crescimento do preço dos combustíveis, principalmente da gasolina. Segunda a Agência Nacional do Petróleo, Gás Natural e Biocombustíveis (ANP), em 2019 no Ceará, o valor do litro da gasolina estava em R \$ 4,344, enquanto em 2014, ano da chegada do Uber no Brasil, a gasolina custava cerca de $\mathrm{R} \$ 3,66$. Tal fato, muitas vezes, se torna uma barreira para quem gostaria de usar o aplicativo como uma alternativa para o desemprego ou aumento de renda.

Diante desses pontos, o estudo em questão se torna ainda mais relevante, principalmente, para pessoas que estão interessadas em iniciar nessa profissão e que, devido à alta no preço da gasolina, podem se desmotivar por não enxergarem um bom investimento. $\mathrm{O}$ artigo traz uma análise comparativa, através do payback, Valor Presente Líquido (VPL) e Taxa Interna de Retorno (TIR), entre a utilização do GNV e da gasolina como forma de evidenciar qual combustível será mais vantajoso economicamente.

O escopo do trabalho será composto por 5 seções: a primeira é a introdução acerca do tema abordado; a segunda evidencia o referencial teórico utilizado no estudo; a terceira apresenta a metodologia abordada; a quarta é composta pelos resultados obtidos e por fim, a quinta, conclui $\mathrm{o}$ artigo com as considerações finais.

\section{Referencial teórico}

Nesta seção, será abordado a teoria utilizada no artigo, através de estudos prévios e de dados. As subseções serão divididas da seguinte maneira:2.1: taxas cobradas pelo Uber sobre as 
corridas, estas serão fundamentais para a obtenção do valor real obtido pelo motorista, seu lucro líquido; 2.2:variação dos preços de mercado da gasolina e do GNV (gás natural veicular), tal análise é o ponto chave de estudo desse artigo, a partir de tais informações será possível analisar o melhor investimento; 2.3: previsão dos valores da gasolina e do GNV para o período analisado, utilizando os dados obtidos no ponto anterior será formulado um valor teórico do preço dos combustíveis para ser utilizado na análise de investimento; 2.4: rendimento dos combustíveis, esses dados também serão de extrema relevância para ter conhecimento da vantagem da troca, ou não, da gasolina pelo GNV e quanto isso impactará financeiramente;2.5 custos de instalação e manutenção do GNV; a partir desses dados, será possível mensurar custos adicionais no processo de mudança; 2.6 análise de investimento e seus indicadores, por meio desses pontos o resultado quantitativo será obtido e ficará claro o impacto do estudo.

\subsection{Taxas cobradas pelo Uber sobre as corridas}

Tabela 1 - Valores das taxas

\begin{tabular}{cccc}
\hline Ano & Preço base & Custo por quilômetro & Custo por minuto \\
\hline 2017 & $\mathrm{R} \$ 2,50$ & $\mathrm{R} \$ 1,20$ & $\mathrm{R} \$ 0,20$ \\
2018 & $\mathrm{R} \$ 1,87$ & $\mathrm{R} \$ 0,71$ & $\mathrm{R} \$ 0,14$ \\
2019 & $\mathrm{R} \$ 1,88$ & $\mathrm{R} \$ 0,90$ & $\mathrm{R} \$ 0,15$ \\
\hline
\end{tabular}

Fonte: Autoria Própria (2019)

\subsection{Variação dos preços de mercado da gasolina e do GNV}

Através de dados divulgados pela ANP - Agência Nacional do Petróleo, Gás Natural e Biocombustíveis - a média de preços anuais no Ceará, de 2017 a 2019, da gasolina, em litros, e do GNV, em metros cúbicos, são:

Tabela 2 - Preços para a gasolina

\begin{tabular}{ll}
\hline Ano & Valor \\
\hline 2017 & $\mathrm{R} \$ 4,170$ \\
2018 & $\mathrm{R} \$ 4,615$ \\
2019 & $\mathrm{R} \$ 4,344$ \\
\hline
\end{tabular}

Fonte: Autoria própria (2019) 
Tabela 3 - Preços para o GNV

\begin{tabular}{ll}
\hline Ano & Valor \\
\hline 2017 & $\mathrm{R} \$ 2,841$ \\
2018 & $\mathrm{R} \$ 3,248$ \\
2019 & $\mathrm{R} \$ 3,400$ \\
\hline
\end{tabular}

Fonte: Autoria própria (2019)

\subsection{Previsão dos valores da gasolina e do GNV}

Obtidos os valores médios do litro da gasolina e do metro cúbico do GNV, nos três últimos anos e através do método quantitativo, Média Móvel Ponderada Simples, serão previstos os valores para os próximos 5 anos, tempo da análise do investimento, dos combustíveis.

Para apoiar decisões as empresas procuram criar sistemas e procedimentos a fim de explorar cenários, com base em informações quantitativas e/ou qualitativa (PASSARI, 2003). Enquanto os métodos qualitativos baseiam-se em opiniões de especialistas, sendo vulneráveis a tendências que podem comprometer a confiabilidade de seus resultados (PELLEGRINI, 2000), os métodos quantitativos utilizam-se basicamente de dados históricos para detectar padrões de comportamento e estimá-los no futuro. Tais modelos empregam ferramental matemáticoestatístico para representar a realidade para a qual foram criados.

No caso em questão será utilizada a Média Móvel Ponderada Simples (MMPS), que consiste na base da Média Móvel, ou seja, uma média dos valores históricos. Todavia, na MMPS é posto um peso em cada valor, normalmente o peso é maior nos períodos mais próximos ao previsto.

$$
X t=\frac{\sum_{i=1}^{n} \quad C i \cdot(X(t-i)}{n}
$$

Para os cálculos propostos, a média será feita com os três últimos valores antecessores ao da previsão e os pesos serão de 0,5 para o valor do ano anterior ao previsto, 0,3 ao intermediário e 0,2 ao valor mais distante.

\subsection{Rendimento dos combustíveis}


Evidenciado na revista Exame, em 2018, através de uma simulação feita na PUC - SP (Pontifícia Universidade Católica de São Paulo) pelo professor de finanças Fábio Gallo, podese analisar a comparação entre a rentabilidade da gasolina e do GNV:

Tabela 4 - Rendimento dos combustíveis

\begin{tabular}{ll}
\hline Combustível & Consumo \\
\hline Gasolina & $10 \mathrm{Km} / \mathrm{litro}$ \\
GNV & $13 \mathrm{~km} / \mathrm{m} 3$ \\
\hline
\end{tabular}

Fonte: Autoria Própria (2019)

\subsection{Custos de instalação e manutenção do GNV}

O investimento inicial para a obtenção de um kit gás $5^{\text {a }}$ geração certificado pelo Inmetro, que é o modelo mais moderno e que perde menos eficiência do carro, está com um valor médio de mercado de $\mathrm{R} \$ 4.800,00$. Entra também na conta a taxa anual cobrada pelo Detran-CE, que tem um valor de $\mathrm{R} \$ 200,00$.

\subsection{Análise de investimento e seus indicadores}

Para a análise da viabilidade econômica, serão utilizados alguns métodos e critérios. Entre os vários existentes, podemos citar: payback simples, payback descontado, VPL (valor presente líquido) e TIR (taxa interna de retorno).

\subsubsection{Payback simples}

É definido como a quantidade de tempo para se recuperar o investimento inicial. O tempo utilizado poderá ser em ano, meses, semanas, dias e etc. Para se calcular esse indicador basta somar todas as entradas de caixa até que a soma desse valor se iguale ao investimento inicial.

\subsubsection{Payback descontado}


O payback descontado leva em consideração o valor do dinheiro no tempo, atualiza os fluxos futuros de caixa a uma taxa de desconto, trazendo os fluxos a valor presente, para depois calcular o período de recuperação (BRUNI, 2008).

Segundo Assaf (2011, p. 379) o cálculo do payback descontado é feito da seguinte forma: “deve-se primeiro trazer cada uma das entradas de caixa a valor presente, descontando esses fluxos a uma taxa de juros que represente a rentabilidade mínima (custo de oportunidade) exigida pela empresa na aceitação do projeto".

\subsubsection{Valor presente líquido (VPL)}

O cálculo da obtenção do Valor Presente Líquido (VPL) é alcançado pela diferença entre o valor presente dos benefícios líquidos de caixa, previsto para cada período de duração do projeto, e o valor presente do investimento (ASSAF NETO e LIMA, 2011).

Tabela 5 - Critério de decisão com o VPL

\begin{tabular}{lll}
\hline VPL > 0 & VPL $=\mathbf{0}$ & VPL $<\mathbf{0}$ \\
\hline Projeto gera valor & Projeto não gera valor & Projeto destrói valor \\
econômico. Cria riqueza & econômico. Não cria & econômico. Reduz riqueza \\
aos acionistas. & riqueza aos acionistas. & dos acionistas. \\
\hline
\end{tabular}

Fonte: Autoria Própria (2019)

Segundo Brealey, Myers e Allen (2013), o Valor Presente Líquido é um método confiável de análise de investimento pois leva em conta unicamente o custo de oportunidade de capital e os fluxos de caixas estimáveis, considerando o valor, ao longo do tempo, do dinheiro. Para os autores, seja qual for o critério de análise de investimento que não considerem o valor temporal do dinheiro, não são adequados.

\subsubsection{Taxa interna de retorno (TIR)}

A taxa interna de retorno é a taxa de juros que iguala o valor presente das entradas de caixa ao valor presente das saídas de caixa. A Taxa Interna de retorno de um investimento é o percentual de retorno alcançado em relação ao saldo do capital investido e ainda não recuperado. (SANTOS, 2001). 


\section{Procedimentos metodológicos}

O estudo realizado tem caráter exploratório, baseado em pesquisas, documentos e bibliografias. Vale salientar que a pesquisa exploratória frequentemente assume a forma de estudo de caso, considerando-se o objetivo do pesquisador em se habituar ao problema e torná-lo acessível. Dessa maneira, o estudo aqui foi empregado para um motorista de aplicativo, do estado do Ceará, que têm duas opções de investimento, a primeira sendo na aquisição de um carro à gasolina e a segunda a aquisição de um carro que tem como forma de combustível alternativa o GNV(gás natural veicular).

Primeiramente, foram coletados dados através de pesquisa de mercado, dados fornecidos pelo DETRAN-CE e pela Secretaria da fazenda, que são:

a) Valor de um carro utilitário (Renault Sandero): $\mathrm{R} \$ 47.990$;

b) Autonomia média da gasolina: $10 \mathrm{Km} / \mathrm{L}$;

c) Autonomia média do gás: $13 \mathrm{Km} / \mathrm{m} 3$;

d) Custo instalação do gás: $\mathrm{R} \$ 4.800,00$;

e) Custo anual de regulação do gás: $\mathrm{R} \$ 200,00$;

f) IPVA, Licenciamento e DPVAT: R\$ 1428,9;

g) Vida útil do carro: 5 anos;

h) Depreciação do carro à gasolina e à gás: R \$ 4.799,00;

i) Alíquota do imposto de renda: $15 \%$.

Como já foi explicado anteriormente, será utilizado o método quantitativo, Média Móvel Ponderada Simples. Com isso, serão previstos os valores para os próximos anos, da gasolina e do GNV:

Tabela 6 - Valores previstos para a gasolina

\begin{tabular}{cc}
\hline Ano & Valor \\
\hline 2019 & $\mathrm{R} \$ 4,153$
\end{tabular}


Fonte: Autoria Própria (2019)

Tabela 7 - Valores previstos para o GNV

\begin{tabular}{ll}
\hline Ano & Valor \\
\hline 2019 & $\mathrm{R} \$ 3,380$ \\
2020 & $\mathrm{R} \$ 3,240$ \\
2021 & $\mathrm{R} \$ 3,291$ \\
2022 & $\mathrm{R} \$ 3,298$ \\
2023 & $\mathrm{R} \$ 3,285$ \\
\hline
\end{tabular}

Fonte: Autoria Própria (2019)

Posteriormente, foi feito uma pesquisa mediante ao aplicativo da UBER para motoristas, com a finalidade de serem encontrados dados para obtenção da média da remuneração diária do motorista:

Tabela 8 - Remunerações e quilometragem rodada por dia

$\overline{\text { Remuneração por quilômetro Remuneração por minuto Média de quilômetros por dia }}$

\begin{tabular}{lll}
\hline $\mathrm{R} \$ 0,90$ & $\mathrm{R} \$ 0,15$ & 142,50
\end{tabular}

Fonte: Autoria Própria (2019)

Então, através de dados obtidos de 7 corridas, que, detalhadas com tempo de viagem, custo de viagem e distância percorrida por viagem, foi calculado uma média de cada um desses parâmetros: 
Tabela 9 - Dados acerca da viagem

$\overline{\text { Distância média por viagem Tempo médio por viagem Remuneração média por viagem }}$

\begin{tabular}{lll}
\hline $\mathrm{R} \$ 0,90$ & $\mathrm{R} \$ 0,15$ & 142,50
\end{tabular}

Fonte: Autoria Própria (2019)

Considerando a média diária de trabalho de um motorista do aplicativo, que é de 9 horas, durante 22 dias úteis por mês, será possível a determinação dos ganhos de despesas diárias, mensais e anuais para os motoristas nos próximos anos, considerando a mudança nos preços dos combustíveis:

Tabela 10 - Parâmetros diários

\begin{tabular}{llll}
\hline Ano & Ganhos diários & $\begin{array}{l}\text { Despesas diárias } \\
\text { (gasolina) }\end{array}$ & $\begin{array}{l}\text { Despesas } \\
\text { diárias (gás) }\end{array}$ \\
\hline 2019 & $\mathrm{R} \$ 212,68$ & $\mathrm{R} \$ 62,57$ & $\mathrm{R} \$ 35,52$ \\
2020 & $\mathrm{R} \$ 168,11$ & $\mathrm{R} \$ 62,57$ & $\mathrm{R} \$ 35,52$ \\
2021 & $\mathrm{R} \$ 183,73$ & $\mathrm{R} \$ 63,00$ & $\mathrm{R} \$ 36,07$ \\
2022 & $\mathrm{R} \$ 179,93$ & $\mathrm{R} \$ 62,57$ & $\mathrm{R} \$ 36,07$ \\
2023 & $\mathrm{R} \$ 181,39$ & $\mathrm{R} \$ 62,71$ & $\mathrm{R} \$ 35,96$ \\
\hline
\end{tabular}

Fonte: Autoria Própria (2019)

Tabela 11 - Parâmetros mensais

\begin{tabular}{llll}
\hline Ano & Ganhos mensais & $\begin{array}{l}\text { Despesas mensais } \\
\text { (gasolina) }\end{array}$ & $\begin{array}{l}\text { Despesas } \\
\text { mensais (gás) }\end{array}$ \\
\hline 2019 & $\mathrm{R} \$ 4.678,92$ & $\mathrm{R} \$ 1.385,92$ & $\mathrm{R} \$ 815,25$ \\
2020 & $\mathrm{R} \$ 3.698,43$ & $\mathrm{R} \$ 1.376,92$ & $\mathrm{R} \$ 781,48$ \\
2021 & $\mathrm{R} \$ 4.042,07$ & $\mathrm{R} \$ 1.385,92$ & $\mathrm{R} \$ 793,54$ \\
2022 & $\mathrm{R} \$ 3.958,37$ & $\mathrm{R} \$ 1.376,52$ & $\mathrm{R} \$ 793,54$ \\
2023 & $\mathrm{R} \$ 3.990,60$ & $\mathrm{R} \$ 1.379,65$ & $\mathrm{R} \$ 791,13$ \\
\hline
\end{tabular}

Fonte: Autoria Própria (2019)

Tabela 12 - Parâmetros anuais 


\begin{tabular}{llll}
\hline Ano & Ganhos anuais & $\begin{array}{l}\text { Despesas anuais } \\
\text { (gasolina) }\end{array}$ & $\begin{array}{l}\text { Despesas anuais } \\
\text { (gás) }\end{array}$ \\
\hline 2019 & $\mathrm{R} \$ 56.147,09$ & $\mathrm{R} \$ 16.631,09$ & $\mathrm{R} \$ 9.782,99$ \\
2020 & $\mathrm{R} \$ 44.381,16$ & $\mathrm{R} \$ 16.518,21$ & $\mathrm{R} \$ 9.377,78$ \\
2021 & $\mathrm{R} \$ 48.504,85$ & $\mathrm{R} \$ 16,631,09$ & $\mathrm{R} \$ 9.522,50$ \\
2022 & $\mathrm{R} \$ 47.500,44$ & $\mathrm{R} \$ 16.518,21$ & $\mathrm{R} \$ 9.522,50$ \\
2023 & $\mathrm{R} \$ 47.887,23$ & $\mathrm{R} \$ 16.555,83$ & $\mathrm{R} \$ 9.493,55$ \\
\hline
\end{tabular}

Fonte: Autoria Própria (2019)

\section{Resultados}

Com o uso do método supracitado, formulou-se os seguintes resultados do exercício para um motorista que em 2019 optasse pelo modelo de carro proposto nas condições citadas de pagamento à vista do valor de $\mathrm{R} \$ 47.990,00$ para o carro sem acessórios e para o valor do carro adicionado do custo de adição dos equipamentos para gás no valor de R $\$ 52.790,00$.

Figura 1 - Resultado do exercício de carro à gasolina.

\begin{tabular}{l|r|r|r|r|r|r}
\hline GASOLINA & 0 & 1 & 2 & 3 & 4 & 5 \\
\hline (+) Receitas & & $R \$ 56.147,09$ & $R \$ 44.381,16$ & $R \$ 48.504,85$ & $R \$ 47.500,44$ & $R \$ 47.887,23$ \\
\hline (-) CV e DV & & $-R \$ 16.631,09$ & $-R \$ 16.518,21$ & $-R \$ 16.631,09$ & $-R \$ 16.518,21$ & $-R \$ 16.555,83$ \\
\hline (-) CF e DF & & $-R \$ 2.428,98$ & $-R \$ 2.428,98$ & $-R \$ 2.428,98$ & $-R \$ 2.428,98$ & $-R \$ 2.428,98$ \\
\hline$(-)$ Deprec & & $-R \$ 4.799,00$ & $-R \$ 4.799,00$ & $-R \$ 4.799,00$ & $-R \$ 4.799,00$ & $-R \$ 4.799,00$ \\
\hline$(=)$ LLAIR & & $R \$ 32.288,02$ & $R \$ 20.634,97$ & $R \$ 24.645,78$ & $R \$ 23.754,25$ & $R \$ 24.103,42$ \\
\hline$(-)$ IR & & $-R \$ 4.843,20$ & $-R \$ 1.547,62$ & $-R \$ 1.848,43$ & $-R \$ 1.781,57$ & $-R \$ 1.807,76$ \\
\hline$(=)$ LLOpDIR & & $R \$ 27.444,82$ & $R \$ 19.087,35$ & $R \$ 22.797,35$ & $R \$ 21.972,68$ & $R \$ 22.295,66$ \\
\hline$(+)$ Deprec. & & $R \$ 4.799,00$ & $R \$ 4.799,00$ & $R \$ 4.799,00$ & $R \$ 4.799,00$ & $R \$ 4.799,00$ \\
\hline$(=)$ FCOp & & $R \$ 32.243,82$ & $R \$ 23.886,35$ & $R \$ 27.596,35$ & $R \$ 26.771,68$ & $R \$ 27.094,66$ \\
\hline (+l-) Investim./desinv. líq.em equip. & $-R \$ 47.990,00$ & & & & & \\
\hline (+l-) Investim./desinv. em cap. de giro & & & & & & \\
\hline$(=)$ FCLivre & $-R \$ 47.990,00$ & $R \$ 32.243,82$ & $R \$ 23.886,35$ & $R \$ 27.596,35$ & $R \$ 26.771,68$ & $R \$ 27.094,66$ \\
\hline
\end{tabular}

Fonte: Autoria Própria (2019)

Figura 2 - Resultado do exercício de carro à gás. 


\begin{tabular}{|l|r|r|r|r|r|r|}
\hline GÁs & 0 & 1 & 2 & 3 & 4 & 5 \\
\hline$(+)$ Receitas & & $R \$ 56.147,09$ & $R \$ 44.381,16$ & $R \$ 48.504,85$ & $R \$ 47.500,44$ & $R \$ 47.887,23$ \\
\hline$(-)$ CV e DV & & $-R \$ 9.782,99$ & $-R \$ 9.377,78$ & $-R \$ 9.522,50$ & $-R \$ 9.522,50$ & $-R \$ 9.493,55$ \\
\hline$(-)$ CF e DF & & $-R \$ 4.128,98$ & $-R \$ 4.128,98$ & $-R \$ 4.128,98$ & $-R \$ 4.128,98$ & $-R \$ 4.128,98$ \\
\hline$(-)$ Deprec & & $-R \$ 4.799,00$ & $-R \$ 4.799,00$ & $-R \$ 4.799,00$ & $-R \$ 4.799,00$ & $-R \$ 4.799,00$ \\
\hline$(=)$ LLAIR & & $R \$ 37.436,12$ & $R \$ 26.075,40$ & $R \$ 30.054,37$ & $R \$ 29.049,96$ & $R \$ 29.465,69$ \\
\hline$(-)$ IR & & $-R \$ 5.615,42$ & $-R \$ 3.911,31$ & $-R \$ 4.508,16$ & $-R \$ 4.357,49$ & $-R \$ 4.419,85$ \\
\hline$(=)$ LLOpDIR & & $R \$ 31.820,70$ & $R \$ 22.164,09$ & $R \$ 25.546,21$ & $R \$ 24.692,47$ & $R \$ 25.045,84$ \\
\hline$(+)$ Deprec. & & $R \$ 4.799,00$ & $R \$ 4.799,00$ & $R \$ 4.799,00$ & $R \$ 4.799,00$ & $R \$ 4.799,00$ \\
\hline$(=)$ FCOp & & $R \$ 36.619,70$ & $R \$ 26.963,09$ & $R \$ 30.345,21$ & $R \$ 29.491,47$ & $R \$ 29.844,84$ \\
\hline$(+$-) Investim./desinv. líq.em equip. & $-R \$ 52.790,00$ & & & & & \\
\hline$(+$ - $)$ Investim./desinv. em cap. de giro & & & & & & \\
\hline$(=)$ FCLivre & $-R \$ 52.790,00$ & $R \$ 36.619,70$ & $R \$ 26.963,09$ & $R \$ 30.345,21$ & $R \$ 29.491,47$ & $R \$ 29.844,84$ \\
\hline
\end{tabular}

Fonte: Autoria Própria (2019)

Tendo em vista que apenas o exercício não representa os indicadores, fez a seguinte tabela comparativa entre payback, VPL e TIR entre os dois modelos, adotando-se um Custo de Capital igual a SELIC de 5\%, como também, abaixo, fez-se um gráfico representativo do VPL para os dois modelos:

Tabela 13 - Comparativo de indicadores

\begin{tabular}{lll}
\hline Item & Gasolina & Gás \\
\hline Custo de capital (a.a) & $5 \%$ & $5 \%$ \\
Payback (anos) & & \\
& 1,70 & 1,60 \\
Payback descontado (anos) & 1,85 & 1,73 \\
VPL (R\$) & $65.594,68$ & $80.402,47$ \\
TRI (\%) & $49,01 \%$ & $53,11 \%$ \\
\hline
\end{tabular}

Fonte: Autoria Própria (2019)

Figura 3 - VPL para o período 


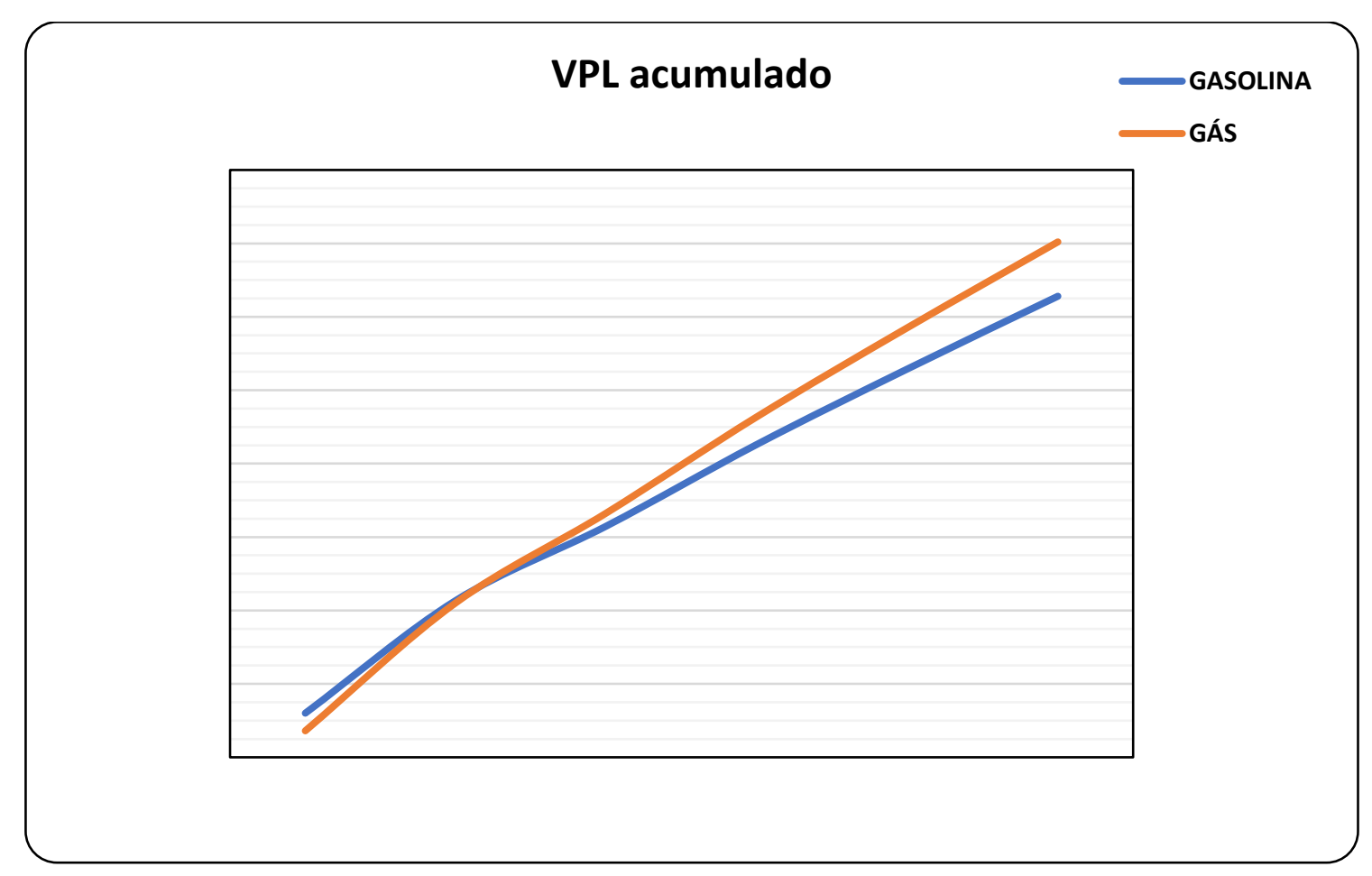

Fonte: Autoria própria (2019)

Com esses resultados em mãos, observa-se as seguintes relações entre as duas opções:

a) Payback descontado da opção de gasolina ocorre mais lento com um resultado de 1,85 anos frente aos 1,73 anos do gás;

b) O Valor Presente Líquido (VPL)do gás ser melhor para o período de 5 anos, com R\$ 80.402,47 frente aos $\mathrm{R} \$ 65.594,68$ da opção de gasolina;

c) A Taxa interna de Retorno do gás, com uma TIR de 53,11\% frente os 49,01\% da opção a gasolina também é uma melhor.

Ainda como é demonstrado no gráfico do VPL para o período, o carro à gás já começa a apresentar melhores resultados que o carro a gasolina antes de completar 2 anos de uso, com um resultado acumulado a partir desse período que apenas reforça essa condição.

\section{Conclusão}

De acordo com os parâmetros analisados nesse trabalho foi possível concluir que a utilização de GNV, em comparação com a gasolina, para motoristas do aplicativo de mobilidade UBER, tem maior viabilidade econômica. A partir dos resultados apresentados, nota-se que o carro movido a GNV tem um Valor Presente Líquido (VPL) maior, payback simples e descontado 
mais rápidos, além de uma Taxa Interna de Retorno (TIR) maior. Dessa forma, um motorista que busque um retorno do investimento para ingressar nessa profissão, deve optar por um carro abastecido de GNV. Ademais, com os parâmetros analisados conclui-se que em menos de 2 anos o investimento teria seu payback tanto para o carro simples, quanto para o carro com acessórios.

Dado a importância da temática, tornava-se necessário o estudo de viabilidade econômica para essa nova classe de trabalhadores. Desse modo, o trabalho cumpriu com o objetivo de analisar ferramentas financeiras, baseado em dados reais, para que esses tenham maior confiança na tomada de decisão.

\section{REFERÊNCIAS}

ASSAF NETO, A; SILVA, C. A. T. Administração do capital de giro. 4. ed. São Paulo: Atlas, 2011.

BAZANI, Adamo. Pesquisa internacional coloca sistemas de transportes públicos de cidades brasileiras entre os piores do mundo. Diário do Transporte, 2018. Disponível em: <https://diariodotransporte.com.br/2018/07/17/pesquisa-internacional-coloca-sistemas-de-transportes-publicosde-cidades-brasileiras-entre-os-piores-do-mundo/>. Acesso em: 10 de nov de 2019.

BREALEY, R. A; MYERS, S. C; ALLEN, F. Princípios de finanças corporativas. 10. ed. Porto Alegre: AMGH, 2013.

BRUNI, A. L. Avaliação de investimento. São Paulo: Atlas, 2008.

PASSARI, A. F. L. Exploração de dados automatizados para a previsão de vendas no varejo utilizando redes neurais. Tese de M.Sc., USP: São Paulo, SP, Brasil, 2005.

PELLEGRINI, F. R. Metodologia para implementação de sistemas de previsão de demanda. Tese de M.Sc., UFRGS: Porto Alegre, RS, Brasil, 2000.

SANTOS, E. O dos. Administração financeira da pequena e média empresa. São Paulo: Atlas, 2001.

UBER. Fatos e Dados sobre a UBER. UBER Newsroom, 2019. Disponível em: <https://www.uber.com/ptBR/newsroom/fatos-e-dados-sobre-uber/>. Acesso em: 10 de nov. de 2019. 